\title{
Finite-time synchronization of uncertain complex dynamic networks with time-varying delay
}

\author{
Yiping Luo ${ }^{1 *}(\mathbb{D}$ and Yuejie Yao
}

\author{
"Correspondence: lyp@hnie.edu.cn \\ ${ }^{1}$ Hunan Institute of Engineering, \\ Xiangtan, China
}

\begin{abstract}
This study investigates the finite-time synchronization of uncertain nonlinear complex dynamic networks with time-varying delay. For a class of complex network models with time-varying delay and uncertain system parameters, the time delay changes infrequently, uncertain terms are unknown but bounded, and the matching conditions are satisfied. The coupling relationship between nodes is a nonlinear function with time delay, and the function satisfies the Lipschitz condition. A new criterion for the finite-time synchronization of a class of complex dynamical networks with variable delay is obtained, and the upper bound of the time for the system to achieve synchronization is presented by constructing a suitable Lyapunov-Krasovskii function, designing a nonlinear controller, and combining analysis techniques, such as matrix inequality. Finally, the validity of finite-time synchronization is verified through computer simulation.
\end{abstract}

Keywords: Complex network; Time-varying delay; Uncertainty; Nonlinear coupling; Finite-time synchronization

\section{Introduction}

Many real-world systems, such as a communication system that consists of opulent interacting, interdependent components that are used to accomplish a unified goal of transmitting and receiving signals, can be described using complex network models. This system is considered as a complex network, and the units that compose the entire system is regarded as the nodes of the complex network. The introduction of a complex network model provides a huge convenience for people to solve many practical problems and has been extensively investigated [1-3]. Synchronization, as a major feature of complex networks, has attracted considerable attention. At present, many different styles of synchronization, such as complete [4], global [5], cluster [6], lag [7], exponential [8], and finite-time synchronization [9], are available.

Delays regularly occur in complex networks given the limited speed of signal transformation or other factors. Various phenomena, such as gravitational time delay, signal propagation over long lines, and human stress response, are inevitable in practical complex dynamic networks. Therefore, the study of synchronization in complex networks must focus

(c) The Author(s) 2020. This article is licensed under a Creative Commons Attribution 4.0 International License, which permits use, sharing, adaptation, distribution and reproduction in any medium or format, as long as you give appropriate credit to the original author(s) and the source, provide a link to the Creative Commons licence, and indicate if changes were made. The images or other third party material in this article are included in the article's Creative Commons licence, unless indicated otherwise in a credit line to the material. If material is not included in the article's Creative Commons licence and your intended use is not permitted by statutory regulation or exceeds the permitted use, you will need to obtain permission directly from the copyright holder. To view a copy of this licence, visit http://creativecommons.org/licenses/by/4.0/. 
on delays to ensure the stability of a system. Many studies on time delay have been recently conducted. The fractional-order synchronization of complex network systems subjected to coupling delay was investigated [10]. Sun et al. [11] theoretically analyzed the feasibility of a complex dynamic network with time delay and discontinuous internal coupling relationship to achieve synchronization. Ma et al. [12] proposed several sufficient conditions that can facilitate synchronization of a single complex network with Markovian jump and time-varying delay by using a pinning control strategy. Wang et al. [13] obtained sufficient conditions for the synchronization of complex systems with time-varying delay by adopting the pinning control strategy.

In practical applications, the system is often expected to reach the synchronous state in a short time. In terms of synchronization speed, exponential synchronization, which can only be synchronized in an infinite time, has received widespread attention due to its fast convergence speed. However, the application of the system will be limited when the system can only be synchronized within an infinite time. Thus, finite-time synchronization of complex networks has attracted considerable attention in recent years. Many studies have been conducted recently on finite-time synchronization of complex networks with time delay. Li et al. [14] realized the synchronization of finite and fixed time delays of a complex dynamic network simultaneously by designing a new controller and using aperiodic intermittent control and obtained several sufficient conditions for system synchronization through eigenvalue characterization. Zhang and Shen et al. [15] proposed several new criteria that can ensure multiple complex systems to achieve finite-time synchronization under periodic or aperiodic discontinuous controllers. Li et al. [16] obtained several sufficient conditions for finite-time synchronization of nonlinearly coupled networks with time-varying delay by performing a new analysis. The finite-time non-fragile synchronization of stochastic complex networks was evaluated in [17]. Xiao and Gan [18] used a continuous finite-time controller and combined linear feedback with finite-time control theory to accomplish the finite-time synchronization of a complex dynamic network with delay.

Except for time-varying delay, fluctuation of system parameters, interference of the external environment, and other phenomena are common and unavoidable, thereby possibly affecting the synchronization performance and stability of a system. In addition, the difference between component and standard parameters may cause changes in other data. Therefore, the study of an uncertain system has huge theoretical importance and much practical value. Few results have been obtained on this issue. For example, in [19], the unknown disturbance and coupling delay were analyzed by employing fractional-order lowpass filter and Luenberger-type state observer. Reference [20] solved the robust finite-time synchronization of an uncertain Markovian complex dynamic network with time-varying delay and reaction-diffusion terms. By designing a feedback controller with update law, Mei [21] evaluated a class of finite-time synchronization problems of a drive-response system that can identify topology structure and uncertain parameters simultaneously and obtained a sufficient condition that can ensure a system to achieve synchronization.

Collectively, the finite-time synchronization of uncertain complex dynamical networks with time-varying delay must be investigated in theoretical and practical applications. Few studies have considered the time-varying delay and uncertainty of a system simultaneously. Zhao and Zheng [22] obtained several new results on finite-time parameter identification and synchronization problems of an uncertain complex dynamic network with 
disturbance and constant time delay. Wang et al. [23] obtained several sufficient conditions for an uncertain complex network with delay by treating many coupling terms as zero terms to attain a finite-time chaotic synchronization state. Reference [24] combined finitetime robust tracking theory with composite nonlinear feedback control and obtained a new criterion of a complex network with external disturbance, parametric uncertainty, and delay to achieve finite-time synchronization. Chaotic systems are considered in Ref. [23, 24]. Yang et al. [25] assessed various finite-time cluster synchronization problems of a T-S fuzzy complex network with nonlinear and probabilistic coupling delays. Zhang and Han [26] realized the finite-time synchronization of two complex networks with different poles, time-varying coupling delays, uncertain parameters, and uncertain topology through a special method of unilateral coupling control; however, the coupling in the investigated system was linear.

Given the above-mentioned analysis, the present study investigates the finite-time synchronization problems for uncertain complex dynamical networks with time-varying delays. Considering the nonlinear internal coupling of nodes of complex networks in many practical models, the internal components must be nonlinear when the network nodes are used as running components. Therefore, in this paper, the coupling relationship between nodes in the system is considered as a nonlinear function and satisfies Lipschitz's condition. In addition, the uncertain term contained in the system is unknown bounded, satisfying the matching condition. Several sufficient conditions for the finite-time synchronization of the uncertain complex dynamic network with time-varying delay are obtained by designing practical controllers. Numerical simulation is performed to verify the effectiveness of the proposed method.

The highlights of this study are presented as follows:

1. A new class of complex dynamic network models with finite-time synchronization control is investigated. The uncertainty of the nodes, the nonlinear coupling between different nodes, and the time-varying coupling delay are all included in this model.

2. A non-fragile control method is proposed and new conclusions are obtained on the basis of the new complex network model. That is, some sufficient conditions for the systems are obtained to achieve finite-time synchronization, and the upper bound of the time required to achieve synchronization is also found.

3. For the proposed complex network model with time-varying delay, Jensen's inequality and other analysis techniques are used to estimate the maximum time delay allowed for the synchronization under the condition that the finite-time synchronization of the system is realized and the parameters are known.

The remainder of this paper is organized as follows. The model of an uncertain complex dynamic network with nonlinear coupling and some preliminaries is introduced in Sect. 2. Some sufficient conditions for the system to achieve finite-time synchronization are obtained in Sect. 3. Several numerical examples are given in Sect. 4 to verify the effectiveness of the theoretical results. Finally, a summary is presented in Sect. 5 .

\section{Preliminaries}

An uncertain complex network with time-varying delay is considered as follows:

$$
\dot{x}_{i}(t)=(A+\Delta A(t)) x_{i}(t)+f\left(x_{i}(t)\right)+\sum_{j=1}^{N} c_{i j} g\left(x_{j}(t)\right)+\sum_{j=1}^{N} d_{i j} g\left(x_{j}(t\right.
$$




$$
-\tau(t)))+u_{i}(t), \quad i=1,2, \ldots, N
$$

where $x_{i}(t)=\left(x_{i 1}(t), x_{i 2}(t), \ldots, x_{i n}(t)\right)^{T} \in R^{n}$ is the state variable of the $i$ th node, $f(x) \in R^{n}$ is a continuous differentiable nonlinear vector function describing the dynamic characteristics of nodes, $g(x) \in R^{n}$ is a smooth nonlinear vector function representing the internal coupling between node state variables, $C=\left(c_{i j}\right)_{N \times N}, D=\left(d_{i j}\right)_{N \times N}$ are externally coupling parameters that represent the topology structure and coupling strength of the network. If there is interaction between node $\mathrm{i}$ and node $\mathrm{j}, c_{i j} \neq 0, d_{i j} \neq 0$, otherwise, $c_{i j}=0$, $d_{i j}=0$. In addition, diagonal elements of the matrices $\mathrm{C}$ and $\mathrm{D}$ are defined as follows: $c_{i i}=-\sum_{j=1, i \neq j}^{N} c_{i j}, d_{i i}=-\sum_{j=1, i \neq j}^{N} d_{i j}$.

A is a given constant matrix, and $\Delta A(t)$ is an uncertain matrix but norm bounded, which satisfies

$$
\Delta A(t)=H F(t) E_{1},
$$

where $H, E_{1}$ are known constant matrices with appropriate dimensions, $F(t)$ is unknown time-varying matrix function, and satisfying that $F^{T}(t) F(t) \leq I, I$ is an identity matrix with suitable dimensions.

Remark 1 Many systems can be characterized by model (1) in practical life. For example, the epidemic disease spread model can be represented by model (1). In this model, everyone can be seen as a node in the network, and disease-related factors are regarded as the components of the nodes. The relationship between the components is nonlinear, and uncertainties are found in the parameters of the system. The influence between people is nonlinear. In addition, the spread of a virus from person to person and the onset of individual diseases will have a certain time delay. Owing to the difference in the strength of each person's physical resistance, time delay produced by different individuals is different and can be regarded as time-varying. The nonlinear coupling is ubiquitous in most practical systems. Several scholars have investigated the nonlinear coupling between various factors within the node $[10,15,16,27,28]$.

Remark 2 Existing studies on the complex dynamic network have rarely considered parametric uncertainty and time-varying delay simultaneously. Only certain literature [29-34] has both investigated parametric uncertainty and time-varying delay. For example, Ref. [29] evaluated the exponential synchronization of complex networks with uncertain parameters; Ref. [30] assessed a complex network with uncertain internal coupling matrix; in addition, Ref. [31, 33] investigated a complex network with uncertain delay; Ref. [32] analyzed robust synchronization with unmodeled dynamic behavior functions, and Ref. [34] considered internal and multiple time-varying coupling time delays simultaneously. However, all of the above-mentioned literature on synchronization was based on infinite time.

Definition 1 ([35]) The delayed dynamic networks (1) is said to be synchronized in finite time, if there exists a constant $t^{*}>0$ ( $t^{*}$ depends on the initial state vector value $x(0)=$ $\left.\left(x_{1}^{T}(0), x_{2}^{T}(0), \ldots, x_{N}^{T}(0)\right)^{T}\right)$, for any $t \geq t^{*}$, such that

$$
\lim _{t \rightarrow t^{*}} \sum_{i=1}^{N}\left\|x_{i}(t)-s(t)\right\|=0,
$$




$$
\left\|x_{i}(t)-s(t)\right\|=0, \quad t \geq t^{*}(i=1, \ldots, N)
$$

where $s(t)=\left(s_{1}(t), s_{2}(t), \ldots, s_{n}(t)\right)^{T} \in R^{n}$ is the particular solution of the system $\dot{s}(t)=(A+$ $\Delta A(t)) s(t)+f(s(t))$.

Assume that $e_{i}(t)=x_{i}(t)-s(t)$ is the synchronization error. According to system (1), we can get the error system as follows:

$$
\begin{aligned}
\dot{e}_{i}(t)= & (A+\Delta A(t)) e_{i}(t)+f\left(x_{i}(t)\right)-f(s(t))+\sum_{j=1}^{N} c_{i j} \phi\left(e_{j}(t)\right) \\
& +\sum_{j=1}^{N} d_{i j} \phi\left(e_{j}(t-\tau(t))\right)+u_{i}(t) .
\end{aligned}
$$

Note $\phi\left(e_{j}(t)\right)=g\left(x_{j}(t)\right)-g(s(t)), \phi\left(e_{j}(t-\tau(t))\right)=g\left(x_{j}(t-\tau(t))\right)-g(s(t-\tau(t)))$.

$u_{i}(t)$ is the control input function in model (1). This paper designs a controller that enables system (1) to achieve finite-time synchronization:

$$
\begin{aligned}
u_{i}(t)= & -h_{1} e_{i}(t)-h_{2} \operatorname{sign}\left(e_{i}(t)\right)\left|e_{i}(t)\right|^{\theta}-\left[h_{3}\left(\int_{t-\tau(t)}^{t} e_{i}^{T}(s) S_{i} e_{i}(s) d s\right)^{\frac{1+\theta}{2}}\right. \\
& \left.+h_{4}\left(\tau_{m} \int_{-\tau_{m}}^{0} \int_{t+\vartheta}^{t} e_{i}^{T}(s) Q_{i} e_{i}(s) d s d \vartheta\right)^{\frac{1+\theta}{2}}\right] \cdot \frac{e_{i}(t)}{\left\|e_{i}(t)\right\|^{2}}
\end{aligned}
$$

where $h_{1}, h_{2}, h_{3}, h_{4}$ are positive constants, $\operatorname{sign}\left(e_{i}(t)\right)=\left\{\begin{array}{l}-1, e_{i}(t)<0 \\ 0, e_{i}(t)=0 \\ 1, e_{i}(t)>0\end{array}\right.$

Remark 3 Some literature works have recently investigated such controllers. The finite and fixed time synchronization of complex networks with delay were evaluated in [14]. Finite-time synchronization of multi-layer nonlinear complex networks was achieved by using intermittent feedback control in [15], and linear complex networks were assessed under the same state in [36]. The problem of adaptive finite-time outer synchronization between two complex networks was solved in [37]. Finite-time synchronization of chaotic systems was investigated in [38]. However, system uncertainty is disregarded in the abovementioned literature.

To end this section, we introduce some assumptions and lemmas for the proof of the main results in the paper.

Assumption 1 Here $\tau(t)$ denotes the internal time-varying delay satisfying

$$
0 \leq \tau_{0} \leq \tau(t) \leq \tau_{m}, \quad \mu_{0} \leq \dot{\tau}(t) \leq \mu_{m}<+\infty
$$

Assumption 2 For the vector-valued function $f(t, x(t))$, assume that there exist positive constants $L>0$ such that $f$ satisfies the semi-Lipschitz condition

$$
(y(t)-x(t))^{T}(f(t, y(t))-f(t, x(t))) \leq L(y(t)-x(t))^{T}(y(t)-x(t)) .
$$


Lemma 1 (See [39] Jensen's inequality) For any constant matrix $Z \in R^{n \times n}, Z=Z^{T}>0$, two scalars $g_{2} \geq g_{1}>0$ such that the following integration is well defined, then

$$
-\left(g_{2}-g_{1}\right) \int_{t-g_{2}}^{t-g_{1}} x^{T}(s) Z x(s) d s \leq-\left(\int_{t-g_{2}}^{t-g_{1}} x(s) d s\right)^{T} Z\left(\int_{t-g_{2}}^{t-g_{1}} x(s) d s\right) .
$$

Lemma 2 (See [40]) Assume that $m_{1}, m_{2}, \ldots, m_{N} \geq 0$. It follows that

$$
\sum_{i=1}^{N} m_{i}^{q} \geq\left(\sum_{i=1}^{N} m_{i}\right)^{p}, \quad 0<p \leq 1 .
$$

Lemma 3 (See [41]) Assume that a continuous, positive-definite function $V(t)$ satisfies the following differential inequality:

$$
\dot{V}(t) \leq-\omega V^{\rho}(t), \quad \forall t \geq t_{0}, V\left(t_{0}\right) \geq 0,
$$

where $\omega>0,0<\rho<1$ are constants. Then, for any given $t_{0}, V(t)$ satisfies the following inequality:

$$
\begin{cases}V^{1-\rho}(t) \leq V^{1-\rho}\left(t_{0}\right)-\omega(1-\rho)\left(t-t_{0}\right), & t_{0} \leq t \leq t^{*} \\ V(t)=0, & t \geq t^{*}\end{cases}
$$

with $t^{*}$ given by $t^{*}=t_{0}+\frac{V^{1-\rho}\left(t_{0}\right)}{\omega(1-\rho)}$.

Lemma 4 (See [42] Schur complement) For a given symmetric matrix $S=S^{T}=\left[\begin{array}{cc}S_{11} & S_{12} \\ * & S_{22}\end{array}\right]$, where $S_{11} \in R^{n \times n}$, the following conditions are equivalent:

1. $S<0$;

2. $S_{11}<0, S_{22}-S_{12}^{T} S_{11}^{-1} S_{12}<0$;

3. $S_{22}<0, S_{11}-S_{12} S_{22}^{-1} S_{12}^{T}<0$.

\section{Main results}

This section focuses on the finite-time synchronization of the complex dynamic network (1) with the control input (4), and some sufficient conditions can be obtained from the following theorems to ensure that the time-varying delay system (1) achieves finite-time synchronization.

Theorem 1 If Assumptions 1 and 2 are all established, positive scalars $\zeta_{i 1}, \zeta_{1}$ and $\zeta_{2}$ are achieved, and the positive symmetric matrices $P_{i}, S_{i}$ and $Q_{i}$ are assumed, satisfying

$$
\left[\begin{array}{ccc}
\Omega_{i 1} & 0 & 0 \\
* & \Omega_{i 2} & 0 \\
* & * & -Q_{i}
\end{array}\right]<0
$$

where

$$
\Omega_{i 1}=P_{i} A+A^{T} P_{i}+\zeta_{i 1} P_{i} H H^{T} P_{i}+\frac{1}{\zeta_{i 1}} E_{1}^{T} E_{1}+2 L_{1} \lambda_{\max }\left(P_{i}\right) I+\zeta_{1}\left(\sum_{j=1}^{N} c_{i j}^{2}\right) P_{i} P_{i}^{T}
$$




$$
\begin{aligned}
& +\frac{N L_{2}^{2}}{\zeta_{1}} I+\zeta_{2}\left(\sum_{j=1}^{N} d_{i j}^{2}\right) P_{i} P_{i}^{T}+S_{i}-2 h_{1} P_{i}+\tau_{m}^{2} Q_{i}, \\
\Omega_{i 2}= & \frac{N L_{3}^{2}}{\zeta_{2}} I-(1-\dot{\tau}(t)) S_{i},
\end{aligned}
$$

then the error system (3) can realize synchronization under the action of controller (4) at a finite time $t^{*}$,

$$
t^{*}=\frac{V^{\frac{1-\theta}{2}}(0)}{M(1-\theta)}
$$

where $M=\min \left(h_{2} \frac{\lambda_{\min }(P)}{\lambda_{\max }^{+\frac{\theta}{2}(P)}}, h_{3} \lambda_{\min }(P), h_{4} \lambda_{\min }(P)\right), P=\operatorname{diag}\left(P_{1}, P_{2}, \ldots, P_{N}\right)$.

Proof Construct the Lyapunov-Krasovskii function $V(t)=\sum_{i=1}^{3} V_{i}(t)$, where $V_{1}(t)=$ $\sum_{i=1}^{N} e_{i}^{T}(t) P_{i} e_{i}(t), \quad V_{2}(t)=\sum_{i=1}^{N} \int_{t-\tau(t)}^{t} e_{i}^{T}(s) S_{i} e_{i}(s) d s, \quad V_{3}(t)=\tau_{m} \sum_{i=1}^{N} \int_{-\tau_{m}}^{0} \int_{t+\vartheta}^{t} e_{i}^{T}(s) \times$ $Q_{i} e_{i}(s) d s d \vartheta$.

The derivative of $V$ along the trajectories (3) and the controller (4) is given by

$$
\begin{aligned}
& \dot{V}_{1}(t)= \sum_{i=1}^{N} 2 e_{i}^{T}(t) P_{i} \dot{e}_{i}(t) \\
&= \sum_{i=1}^{N} 2 e_{i}^{T}(t) P_{i}\left[(A+\Delta A(t)) e_{i}(t)+\left(f\left(x_{i}(t)\right)-f(s(t))\right)+\sum_{j=1}^{N} c_{i j} \phi\left(e_{j}(t)\right)\right. \\
&+\sum_{j=1}^{N} d_{i j} \phi\left(e_{j}(t-\tau(t))\right)-h_{1} e_{i}(t)-h_{2} \operatorname{sign}\left(e_{i}(t)\right)\left|e_{i}(t)\right|^{\theta}-\left[h _ { 3 } \left(\int_{t-\tau(t)}^{t} e_{i}^{T}(s)\right.\right. \\
&\left.\left.\left.\times S_{i} e_{i}(s) d s\right)^{\frac{1+\theta}{2}}+h_{4}\left(\tau_{m} \int_{-\tau_{m}}^{0} \int_{t+\vartheta}^{t} e_{i}^{T}(s) Q_{i} e_{i}(s) d s d \vartheta\right)^{\frac{1+\theta}{2}}\right] \cdot \frac{e_{i}(t)}{\left\|e_{i}(t)\right\|^{2}}\right], \\
& 2 e_{i}^{T}(t) P_{i}(A+\Delta A(t)) e_{i}(t)=e_{i}^{T}(t)\left[P_{i} A+A^{T} P_{i}\right] e_{i}(t)+2 e_{i}^{T}(t) P_{i} H F(t) E_{1} e_{i}(t), \\
& 2 e_{i}^{T}(t) P_{i} H F(t) E_{1} e_{i}(t) \leq \zeta_{i 1} e_{i}^{T}(t) P_{i} H H^{T} P_{i}^{T} e_{i}(t)+\frac{1}{\zeta_{i 1}} e_{i}^{T}(t) E_{1}^{T} E_{1} e_{i}(t) .
\end{aligned}
$$

According to Assumption 2, we can get

$$
\begin{aligned}
& 2 e_{i}^{T}(t) P_{i}\left(f\left(x_{i}(t)\right)-f(s(t))\right) \leq 2 L_{1} \lambda_{\max }\left(P_{i}\right) e_{i}^{T}(t) e_{i}(t), \\
& \sum_{i=1}^{N} 2 e_{i}^{T}(t) P_{i} \sum_{j=1}^{N} c_{i j} \phi\left(e_{j}(t)\right) \leq \sum_{i=1}^{N}\left[\zeta_{1} e_{i}^{T}(t)\left(\sum_{j=1}^{N} c_{i j}^{2}\right) P_{i} P_{i}^{T} e_{i}(t)+\frac{N L_{2}^{2}}{\zeta_{1}} e_{i}^{T}(t) e_{i}(t)\right], \\
& \sum_{i=1}^{N} 2 e_{i}^{T}(t) P_{i} u_{i} \\
& =\sum_{i=1}^{N} 2 e_{i}^{T}(t) P_{i}\left[-h_{1} e_{i}(t)-h_{2} \operatorname{sign}\left(e_{i}(t)\right)\left|e_{i}(t)\right|^{\theta}-\left[h _ { 3 } \left(\int_{t-\tau(t)}^{t} e_{i}^{T}(s)\right.\right.\right. \\
& \left.\left.\left.\quad \times S_{i} e_{i}(s) d s\right)^{\frac{1+\theta}{2}}+h_{4}\left(\tau_{m} \int_{-\tau_{m}}^{0} \int_{t+\vartheta}^{t} e_{i}^{T}(s) Q_{i} e_{i}(s) d s d \vartheta\right)^{\frac{1+\theta}{2}}\right] \cdot \frac{e_{i}(t)}{\left\|e_{i}(t)\right\|^{2}}\right]
\end{aligned}
$$


Lu and Yo Advances in Difference Equations

(2020) $2020: 32$

Page 8 of 22

$$
\begin{aligned}
\leq & -2 h_{1} \sum_{i=1}^{N} e_{i}^{T}(t) P_{i} e_{i}(t)-2 \sum_{i=1}^{N}\left[h_{2} \lambda_{\min }\left(P_{i}\right)\left|e_{i}(t)\right|^{\theta+1}+h_{3} \lambda_{\min }\left(P_{i}\right)\left(\int_{t-\tau(t)}^{t} e_{i}^{T}(s)\right.\right. \\
& \left.\left.\times S_{i} e_{i}(s) d s\right)^{\frac{\theta+1}{2}}+h_{4} \lambda_{\min }\left(P_{i}\right)\left(\tau_{m} \int_{-\tau_{m}}^{0} \int_{t+\vartheta}^{t} e_{i}^{T}(s) Q_{i} e_{i}(s) d s d \vartheta\right)^{\frac{1+\theta}{2}}\right] \\
\leq & -2 h_{1} \sum_{i=1}^{N} e_{i}^{T}(t) P_{i} e_{i}(t)-2 M \sum_{i=1}^{N}\left[\left(e_{i}^{T}(t) P_{i} e_{i}(t)\right)^{\frac{\theta+1}{2}}+\left(\int_{t-\tau(t)}^{t} e_{i}^{T}(s) S_{i} e_{i}(s) d s\right)^{\frac{\theta+1}{2}}\right. \\
& \left.+\left(\tau_{m} \int_{-\tau_{m}}^{0} \int_{t+\vartheta}^{t} e_{i}^{T}(s) Q_{i} e_{i}(s) d s d \vartheta\right)^{\frac{1+\theta}{2}}\right]
\end{aligned}
$$

where $M=\min \left(h_{2} \frac{\lambda_{\min }(P)}{\lambda_{\max }^{\frac{1+\theta}{2}}(P)}, h_{3} \lambda_{\min }(P), h_{4} \lambda_{\min }(P)\right)$. We have

$$
\dot{V}_{2}(t)=\sum_{i=1}^{N}\left[e_{i}^{T}(t) S_{i} e_{i}(t)-(1-\dot{\tau}(t)) e_{i}^{T}(t-\tau(t)) S_{i} e_{i}(t-\tau(t))\right]
$$

According to Lemma 1,

$$
\begin{aligned}
& \dot{V}_{3}(t)=\tau_{m}^{2} \sum_{i=N}^{N} e_{i}^{T}(t) Q_{i} e_{i}(t)-\tau_{m} \sum_{i=1}^{N} \int_{t-\tau_{m}}^{t} e_{i}^{T}(s) Q_{i} e_{i}(s) d s \\
& \leq \tau_{m}^{2} \sum_{i=N}^{N} e_{i}^{T}(t) Q_{i} e_{i}(t)-\sum_{i=1}^{N}\left(\int_{t-\tau_{m}}^{t} e_{i}^{T}(s) d s\right)^{T} Q_{i}\left(\int_{t-\tau_{m}}^{t} e_{i}(s) d s\right), \\
& \dot{V}(t) \leq \sum_{i=1}^{N} e_{i}^{T}(t)\left[P_{i} A+A^{T} P_{i}+\zeta_{i 1} P_{i} H H^{T} P_{i}+\frac{1}{\zeta_{i 1}} E_{1}^{T} E_{1}+2 L_{1} \lambda_{\max }\left(P_{i}\right) I+\tau_{m}^{2} Q_{i}\right. \\
& \left.+\zeta_{1}\left(\sum_{j=1}^{N} c_{i j}^{2}\right) P_{i} P_{i}^{T}+\frac{N L_{2}^{2}}{\zeta_{1}} I+\zeta_{2}\left(\sum_{j=1}^{N} d_{i j}^{2}\right) P_{i} P_{i}^{T}+S_{i}-2 h_{1} \lambda_{\min }\left(P_{i}\right) I\right] e_{i}(t) \\
& +\sum_{i=1}^{N} e_{i}^{T}(t-\tau(t))\left[\frac{N L_{3}^{2}}{\zeta_{2}} I-(1-\dot{\tau}(t)) S_{i}\right] e_{i}(t-\tau(t)) \\
& -\sum_{i=1}^{N}\left(\int_{t-\tau_{m}}^{t} e_{i}^{T}(s) d s\right)^{T} Q_{i}\left(\int_{t-\tau_{m}}^{t} e_{i} d s\right)-2 M \sum_{i=1}^{N}\left[\left(e_{i}^{T}(t) P_{i} e_{i}(t)\right)^{\frac{\theta+1}{2}}\right. \\
& \left.+\left(\int_{t-\tau(t)}^{t} e_{i}^{T}(s) S_{i} e_{i}(s) d s\right)^{\frac{\theta+1}{2}}+\left(\tau_{m} \int_{-\tau_{m}}^{0} \int_{t+\vartheta}^{t} e_{i}^{T}(s) Q_{i} e_{i}(s) d s d \vartheta\right)^{\frac{1+\theta}{2}}\right] \\
& =\sum_{i=1}^{N} \xi_{i}^{T}(t) \Omega \xi_{i}(t)-2 M \sum_{i=1}^{N}\left[\left(e_{i}^{T}(t) P_{i} e_{i}(t)\right)^{\frac{\theta+1}{2}}+\left(\int_{t-\tau(t)}^{t} e_{i}^{T}(s) S_{i} e_{i}(s) d s\right)^{\frac{\theta+1}{2}}\right. \\
& \left.+\left(\tau_{m} \int_{-\tau_{m}}^{0} \int_{t+\vartheta}^{t} e_{i}^{T}(s) Q_{i} e_{i}(s) d s d \vartheta\right)^{\frac{1+\theta}{2}}\right], \\
& \xi_{i}(t)=\left[\begin{array}{lll}
e_{i}^{T}(t) & e_{i}^{T}(t-\tau(t)) & \left(\int_{t-\tau_{0}}^{t} e_{i}^{T}(s) d s\right)^{T}
\end{array}\right]^{T}, \quad \Omega=\left[\begin{array}{ccc}
\Omega_{i 1} & 0 & 0 \\
* & \Omega_{i 2} & 0 \\
* & * & -Q_{i}
\end{array}\right],
\end{aligned}
$$




$$
\begin{aligned}
\Omega_{i 1}= & P_{i} A+A^{T} P_{i}+\zeta_{i 1} P_{i} H H^{T} P_{i}+\frac{1}{\zeta_{i 1}} E_{1}^{T} E_{1}+2 L_{1} \lambda_{\max }\left(P_{i}\right) I+\zeta_{1}\left(\sum_{j=1}^{N} c_{i j}^{2}\right) P_{i} P_{i}^{T} \\
& +\frac{N L_{2}^{2}}{\zeta_{1}} I+\zeta_{2}\left(\sum_{j=1}^{N} d_{i j}^{2}\right) P_{i} P_{i}^{T}+S_{i}-2 h_{1} P_{i}+\tau_{m}^{2} Q_{i}, \\
\Omega_{i 2}= & \frac{N L_{3}^{2}}{\zeta_{2}} I-(1-\dot{\tau}(t)) S_{i} .
\end{aligned}
$$

According to Lemma 2,

$$
\begin{aligned}
& \left(e_{i}^{T}(t) P_{i} e_{i}(t)\right)^{\frac{\theta+1}{2}}+\left(\int_{t-\tau(t)}^{t} e_{i}^{T}(s) S_{i} e_{i}(s) d s\right)^{\frac{\theta+1}{2}}+\left(\tau_{m} \int_{-\tau_{m}}^{0} \int_{t+\vartheta}^{t} e_{i}^{T}(s) Q_{i} e_{i}(s) d s d \vartheta\right)^{\frac{1+\theta}{2}} \\
& \geq\left[\left(e_{i}^{T}(t) P_{i} e_{i}(t)\right)+\int_{t-\tau(t)}^{t} e_{i}^{T}(s) S_{i} e_{i}(s) d s+\left(\tau_{m} \int_{-\tau_{m}}^{0} \int_{t+\vartheta}^{t} e_{i}^{T}(s) Q_{i} e_{i}(s) d s d \vartheta\right)\right]^{\frac{\theta+1}{2}} \\
& \dot{V}(t) \leq \sum_{i=1}^{N} \xi_{i}^{T}(t) \Omega \xi_{i}(t)-2 M V^{\frac{\theta+1}{2}}(t) .
\end{aligned}
$$

If the inequality (5) holds, then

$$
\dot{V}(t) \leq-2 M V^{\frac{\theta+1}{2}}(t)
$$

From Lemma 3, we can conclude that the system (1) can reach synchronization in a finite time $t^{*}$, and the time $t^{*}$ is given by

$$
t^{*}=\frac{V^{\frac{1-\theta}{2}}(0)}{M(1-\theta)}
$$

where

$$
V(0)=\sum_{i=1}^{N}\left[e_{i}^{T}(0) P_{i} e_{i}(0)+\int_{-\tau(0)}^{0} e_{i}^{T}(s) S_{i} e_{i}(s) d s+\int_{-\tau_{m}}^{0} \int_{\vartheta}^{0} e_{i}^{T}(s) Q_{i} e_{i}(s) d s d \vartheta\right] .
$$

Remark 4 Numerous achievements on finite-time synchronization of complex networks have been accomplished, and few studies have considered parametric uncertainty and time-varying delay simultaneously. Reference [12] investigated the robust and stochastic finite-time synchronization of Markovian complex network with reaction-diffusion terms. Reference [20] discussed a neural network. For the system model studied in Ref. [21], the internal coupling relationship is linear, which is different from our model and method. Reference [25] evaluated the cluster synchronization of a fuzzy complex network.

Theorem 2 Assume that there are symmetric positive-definite matrices $\tilde{P}_{i}, \tilde{S}_{i}, Q_{i}$ and positive scalars $\zeta_{i 1}, \zeta_{1}, \zeta_{2}$ so that the following inequalities hold:

$$
\left[\begin{array}{ccccc}
\tilde{\Omega}_{i 1} & \tilde{P}_{i} \mathrm{E}_{1}^{T} & \mathrm{~L}_{2} \tilde{P}_{i} & \tilde{P}_{i} & \tau_{m} \tilde{P}_{i} \\
E_{1} \tilde{P}_{i} & -\zeta_{i 1} I & 0 & 0 & 0 \\
\mathrm{~L}_{2} \tilde{P}_{i} & 0 & -\frac{\zeta_{1} I}{N} I & 0 & 0 \\
\tilde{P}_{i} & 0 & 0 & -\tilde{S}_{i} & 0 \\
\tau_{m} \tilde{P}_{i} & 0 & 0 & 0 & -\mathrm{Q}_{i}
\end{array}\right]<0
$$




$$
\begin{aligned}
& {\left[\begin{array}{cc}
-(1-\dot{\tau}(t)) S_{i} & \mathrm{~L}_{3} \tilde{S}_{i} \\
L_{3} \tilde{S}_{i} & -\frac{\zeta_{2}}{N} I
\end{array}\right]<0,} \\
& -Q_{i}<0
\end{aligned}
$$

where

$$
\tilde{\Omega}_{i 1}=A \tilde{P}_{i}+\tilde{P}_{i} A^{T}+\zeta_{i 1} H H^{T}+\frac{2 L_{1} I}{\lambda_{\min }\left(\tilde{P}_{i}\right)}+\zeta_{1}\left(\sum_{j=1}^{N} c_{i j}^{2}\right) I+\zeta_{2}\left(\sum_{j=1}^{N} d_{i j}^{2}\right) I-2 h_{1} \tilde{P}_{i} .
$$

Then uncertain complex networks can achieve finite-time synchronization.

Proof According to Lemma 4, the matrix inequality (5) is equal to the inequalities:

$$
\begin{aligned}
& {\left[\begin{array}{ccccc}
\hat{\Omega}_{i 1} & \mathrm{E}_{1}^{T} & \mathrm{~L}_{2} I & \mathrm{I} & \tau_{m} I \\
E_{1} & -\zeta_{i 1} I & 0 & 0 & 0 \\
\mathrm{~L}_{2} I & 0 & -\frac{\zeta_{1} I}{N} & 0 & 0 \\
I & 0 & 0 & -S_{i}^{-1} & 0 \\
\tau_{m} I & 0 & 0 & 0 & -\mathrm{Q}_{i}
\end{array}\right]<0,} \\
& {\left[\begin{array}{cc}
-(1-\dot{\tau}(t)) S_{i} & \mathrm{~L}_{3} I \\
L_{3} I & -\frac{\zeta_{2}}{N} I
\end{array}\right]<0,} \\
& -Q_{i}<0 .
\end{aligned}
$$

Here $\hat{\Omega}_{i 1}=P_{i} A+A^{T} P_{i}+\zeta_{i 1} P_{i} H H^{T} P_{i}+2 L_{1} \lambda_{\max }\left(P_{i}\right) I+\zeta_{1}\left(\sum_{j=1}^{N} c_{i j}^{2}\right) P_{i} P_{i}^{T}+\zeta_{2}\left(\sum_{j=1}^{N} d_{i j}^{2}\right) P_{i} P_{i}^{T}-$ $2 h_{1} P_{i}$.

Premultiplying and postmultiplying both sides of (9)-(10) by $\operatorname{diag}\left(\left[P_{i}^{-1}, I, I, I, I\right]\right)$, $\operatorname{diag}\left(\left[S_{i}^{-1}, I\right]\right)$, respectively, it is clear that inequalities (9)-(10) are equal to

$$
\begin{aligned}
& {\left[\begin{array}{ccccc}
\check{\Omega}_{i 1} & \mathrm{P}_{i}^{-1} \mathrm{E}_{1}^{T} & \mathrm{~L}_{2} \mathrm{P}_{i}^{-1} & \mathrm{P}_{i}^{-1} & \tau_{m} \mathrm{P}_{i}^{-1} \\
E_{1} \mathrm{P}_{i}^{-1} & -\zeta_{i 1} I & 0 & 0 & 0 \\
\mathrm{~L}_{2} \mathrm{P}_{i}^{-1} & 0 & -\frac{\zeta_{1}}{N} I & 0 & 0 \\
\mathrm{P}_{i}^{-1} & 0 & 0 & -S_{i}^{-1} & 0 \\
\tau_{m} \mathrm{P}_{i}^{-1} & 0 & 0 & 0 & -\mathrm{Q}_{i}
\end{array}\right]<0,} \\
& {\left[\begin{array}{cc}
-(1-\dot{\tau}(t)) S_{i} & \mathrm{~L}_{3} S_{i}^{-1} \\
L_{3} S_{i}^{-1} & -\frac{\zeta_{2} I}{N} I
\end{array}\right]<0,}
\end{aligned}
$$

where $\check{\Omega}_{i 1}=A P_{i}^{-1}+P_{i}^{-1} A^{T}+\zeta_{i 1} H H^{T}+2 L_{1} \lambda_{\max }\left(P_{i}^{-1}\right) I+\zeta_{1}\left(\sum_{j=1}^{N} c_{i j}^{2}\right) I+\zeta_{2}\left(\sum_{j=1}^{N} d_{i j}^{2}\right) I-2 h_{1} P_{i}^{-1}$. Let $P_{i}^{-1}=\tilde{P}_{i}, S_{i}^{-1}=\tilde{S}_{i}$, then (12)-(13) are equal to (6)-(7).

Remark 5 According to Theorem 1, the maximum value of time delay $\tau_{m}$, which ensures finite-time synchronization is realized in the system, can be calculated, and the maximum value can be obtained by optimizing LMI (6). At present, some scholars have studied this problem, but few have applied this method to the problems of finite-time synchronization in complex networks. The extended dissipative synchronization of complex dynamic networks was studied in [43]. Yu [44] proposed a kind of mixed topologies with time delay, 
Shi [45] researched the outer synchronization problem of delayed complex network under pinning control, Wang [46] studied the synchronization problem of two nonidentical complex-valued neural networks, Huang [47] obtained some sufficient conditions for the Markovian jump complex networks to achieve finite-time $H_{\infty}$ sampled-data synchronization. However, this work did not consider the problem of time-delay related finite-time synchronization, which is due to the difficulty of controller design.

In the proof of Theorem 1, for the system (1), if P is an identity matrix when constructing the Lyapunov-Krasovskii function, then the following conclusion can be reached easily.

Corollary 1 Under Assumptions 1 and 2, positive scalars $\varepsilon_{i 1}, \varepsilon_{1}, \varepsilon_{2}$ are known. If there exist positive-definite symmetric matrices $S_{i}$ and $Q_{i}$ satisfy

$$
\left[\begin{array}{ccc}
\Omega_{i 1} & 0 & 0 \\
0 & \Omega_{i 2} & 0 \\
* & * & -Q_{i}
\end{array}\right]<0,
$$

where

$$
\begin{aligned}
\Omega_{i 1}= & A+A^{T}+\varepsilon_{i 1} H H^{T}+\frac{1}{\varepsilon_{i 1}} E_{1}^{T} E_{1}+2 L_{1} I+\varepsilon_{1}\left(\sum_{j=1}^{N} c_{i j}^{2}\right) I+\frac{N L_{2}^{2}}{\varepsilon_{1}} I \\
& +\varepsilon_{2}\left(\sum_{j=1}^{N} d_{i j}^{2}\right) I+S_{i}-2 h_{1} I+\tau_{m}^{2} Q_{i} \\
\Omega_{i 2}= & \frac{N L_{3}^{2}}{\varepsilon_{2}} I-(1-\dot{\tau}(t)) S_{i} .
\end{aligned}
$$

Then the error system (3) can reach synchronization at finite time. The time can be estimated as

$$
t^{*}=\frac{V^{\frac{1-\theta}{2}}(0)}{M(1-\theta)}
$$

where $M=\min \left(h_{2}, h_{3}, h_{4}\right)$.

No uncertainty exists in the system when $\Delta A(t)=0$, and the equation of state at this time is

$$
\begin{aligned}
\dot{x}_{i}(t) & =A x_{i}(t)+f\left(x_{i}(t)\right)+\sum_{j=1}^{N} c_{i j} g\left(x_{j}(t)\right)+\sum_{j=1}^{N} d_{i j} g\left(x_{j}(t-\tau(t))\right)+u_{i}(t), \\
& i=1,2, \ldots, N .
\end{aligned}
$$

The error state function is

$$
\begin{aligned}
\dot{e}_{i}(t)= & A e_{i}(t)+\left(f\left(x_{i}(t)\right)-f(s(t))\right)+\sum_{j=1}^{N} c_{i j} \phi\left(e_{j}(t)\right)+\sum_{j=1}^{N} d_{i j} \phi\left(e_{j}(t\right. \\
& -\tau(t)))+u_{i}(t) .
\end{aligned}
$$


Corollary 2 Suppose that there exist positive-definite matrices $P_{i}, S_{i}$ and $Q_{i}$, positive scalars $\varepsilon_{1}, \varepsilon_{2}$ to make the following inequality holds:

$$
\left[\begin{array}{ccc}
\Omega_{i 1} & 0 & 0 \\
0 & \Omega_{i 2} & 0 \\
* & * & -Q_{i}
\end{array}\right]<0,
$$

where

$$
\begin{aligned}
\Omega_{i 1}= & P_{i} A+A^{T} P_{i}+2 L_{1} \lambda_{\max }\left(P_{i}\right) I+\varepsilon_{1}\left(\sum_{j=1}^{N} c_{i j}^{2}\right) P_{i} P_{i}^{T}+\frac{N L_{2}^{2}}{\varepsilon_{1}} I+\varepsilon_{2}\left(\sum_{j=1}^{N} d_{i j}^{2}\right) P_{i} P_{i}^{T} \\
& +S_{i}-2 h_{1} P_{i}+\tau_{m}^{2} Q_{i}, \\
\Omega_{i 2}= & \frac{N L_{3}^{2}}{\varepsilon_{2}} I-(1-\dot{\tau}(t)) S_{i} .
\end{aligned}
$$

Then we can draw the conclusion that the error system (16) can realize finite-time synchronization. And the settling time

$$
t^{*}=\frac{V^{\frac{1-\theta}{2}}(0)}{M(1-\theta)}
$$

where $M=\min \left(h_{2} \frac{\lambda_{\min }\left(P_{i}\right)}{\lambda_{\max }^{\frac{1+\theta}{2}}(P)}, h_{3} \lambda_{\min }(P), h_{4} \lambda_{\min }(P)\right)$

Remark 6 Finite-time synchronization of complex network models without uncertainties has been investigated and has obtained considerable results. However, these studies are different from the model or method proposed in this paper. For example, in the network model of Ref. [18], the coefficient matrix of the dynamic characteristic function is an identity matrix, and the coupling relationship between the nodes is a linear matrix. Reference [28] evaluated a class of neural networks. Reference [48] assessed unilateral coupling of complex networks. Reference [49] investigated hybrid projective synchronization.

In Theorem 1, we design the controller with indeterminate exponential terms $\theta$, in which only condition $0<\theta<1$ must be satisfied. However, in some cases, we can directly specify the exponential value in the controller, so that we can calculate the time required for the system to achieve synchronization. Especially, when $\theta=0$, the controller takes the following form:

$$
\begin{aligned}
u_{i}(t)= & -h_{1} e_{i}(t)-h_{2} \operatorname{sign}\left(e_{i}(t)\right) \Pi-h_{3}\left(\int_{t-\tau(t)}^{t} e_{i}^{T}(s) S_{i} e_{i}(s) d s\right)^{\frac{1}{2}} \cdot \frac{e_{i}(t)}{\|e(t)\|^{2}} \\
& -h_{4}\left(\tau_{m} \int_{-\tau_{m}}^{0} \int_{t+\vartheta}^{t} e_{i}^{T}(s) Q_{i} e_{i}(s) d s d \vartheta\right)^{\frac{1}{2}} \cdot \frac{e_{i}(t)}{\left\|e_{i}(t)\right\|^{2}}
\end{aligned}
$$

where $\Pi=\underbrace{(1,1, \ldots, 1)^{T}}_{n}$. 
Corollary 3 Under Assumptions 1 and 2 , and when positive scalars $\varepsilon_{i 1}, \varepsilon_{1}$ and $\varepsilon_{2}$ are available, assume that there exist positive-definite symmetric matrices $P_{i}, S_{i}$ and $Q_{i}$, so that

$$
\left[\begin{array}{ccc}
\Omega_{i 1} & 0 & 0 \\
* & \Omega_{i 2} & 0 \\
* & * & -Q_{i}
\end{array}\right]<0
$$

where

$$
\begin{aligned}
\Omega_{i 1}= & P_{i} A+A^{T} P_{i}+\varepsilon_{i 1} P_{i} H H^{T} P_{i}+\frac{1}{\varepsilon_{i 1}} E_{1}^{T} E_{1}+2 L_{1} \lambda_{\min }\left(P_{i}\right) I+\varepsilon_{1}\left(\sum_{j=1}^{N} c_{i j}^{2}\right) P_{i} P_{i}^{T} \\
& +\frac{N L_{2}^{2}}{\varepsilon_{1}} I+\varepsilon_{2}\left(\sum_{j=1}^{N} d_{i j}^{2}\right) P_{i} P_{i}^{T}+S_{i}-2 h_{1} P_{i}+\tau_{m}^{2} Q_{i}, \\
\Omega_{i 2}= & \frac{N L_{3}^{2}}{\varepsilon_{2}} I-(1-\dot{\tau}(t)) S_{i},
\end{aligned}
$$

then the error system (3) can achieve synchronization in finite time under the controller (19):

$$
t^{*}=\frac{V^{\frac{1}{2}}(0)}{M}
$$

where $M=\min \left(h_{2} \frac{\lambda_{\min }(P)}{\lambda_{\max }^{\frac{1}{2}}(P)}, h_{3} \lambda_{\min }(P), h_{4} \lambda_{\min }(P)\right)$.

In the actual application of the system, implementing the controller is affected by different factors, such as hardware and software. Thus, the controller parameters have an uncertain change, and this change causes the performance of the system to decline or destroy the original stability. The system becomes fragile when a traditional controller is used. Thus, we use a non-fragile controller. A good control performance can be maintained under the uncertainty that the controller allows through this method. Then the feasibility of the theory is proven. When the controller is expressed in the following form:

$$
\begin{aligned}
u_{i}(t)= & -\left(K_{i}+\Delta K_{i}(t)\right) e_{i}(t)-h_{1} \operatorname{sign}\left(e_{i}(t)\right) \Pi-\left[h _ { 2 } \left(\int_{t-\tau(t)}^{t} e_{i}^{T}(s) S_{i}\right.\right. \\
& \left.\left.\times e_{i}(s) d s\right)^{\frac{1}{2}}+h_{3}\left(\tau_{m} \int_{-\tau_{m}}^{0} \int_{t+\vartheta}^{t} e_{i}^{T}(s) Q_{i} e_{i}(s) d s d \vartheta\right)^{\frac{1}{2}}\right] \cdot \frac{e_{i}(t)}{\left\|e_{i}(t)\right\|^{2}},
\end{aligned}
$$

where $K_{i} \in R^{n \times n}$ is the constant control gain, $h_{1}, h_{2}, h_{3}>0$,and $\Delta K_{i}(t)$ are the normbounded uncertain matrix of the system, which satisfies $\Delta K(t)=H F(t) E_{2}$, then, for the complex dynamic network (1), we can draw the following conclusion.

Theorem 3 Assume that Assumptions 1 and 2 are established, $\varepsilon_{i 1}, \varepsilon_{i 2}, \varepsilon_{1}$ and $\varepsilon_{2}$ are known positive scalars, and positive-definite matrices $K_{i}, P_{i}, S_{i}$ and $Q_{i}$ exist to satisfy the following 
requirements:

$$
\left[\begin{array}{ccc}
\Omega_{i 1} & 0 & 0 \\
* & \Omega_{i 2} & 0 \\
* & * & -Q_{i}
\end{array}\right]<0
$$

where

$$
\begin{aligned}
\Omega_{i 1}= & P_{i} A+A^{T} P_{i}+\varepsilon_{i 1} P_{i} H H^{T} P_{i}+\frac{1}{\varepsilon_{i 1}} E_{1}^{T} E_{1}+2 L_{1} \lambda_{\max }\left(P_{i}\right) I+\varepsilon_{1}\left(\sum_{j=1}^{N} c_{i j}^{2}\right) P_{i} P_{i}^{T} \\
& +\frac{N L_{2}^{2}}{\varepsilon_{1}} I+\varepsilon_{2}\left(\sum_{j=1}^{N} d_{i j}^{2}\right) P_{i} P_{i}^{T}+S_{i}-2 P_{i} K_{i}+K_{i}^{T} P_{i}+\varepsilon_{i 2} P_{i} H H^{T} P_{i} \\
& +\frac{1}{\varepsilon_{i 2}} E_{2}^{T} E_{2}+\tau_{m}^{2} Q_{i}, \\
\Omega_{i 2}= & \frac{N L_{3}^{2}}{\varepsilon_{2}} I-(1-\dot{\tau}(t)) S_{i},
\end{aligned}
$$

then system (1) is synchronized in a finite time, and the time is calculated as

$$
t^{*}=\frac{V^{\frac{1}{2}}(0)}{W},
$$

where $V(0)=\sum_{i=1}^{N}\left[e_{i}^{T}(0) P_{i} e_{i}(0)+\int_{-\tau(0)}^{0} e_{i}^{T}(s) S_{i} e_{i}(s) d s+\int_{-\tau_{m}}^{0} \int_{\theta}^{0} e_{i}^{T}(s) Q_{i} e_{i}(s) d s d \theta\right]$, and $W=$ $\min \left(h_{1} \frac{\lambda_{\min }(P)}{\lambda_{\max }^{\frac{1}{2}}(P)}, h_{2} \lambda_{\min }(P), h_{3} \lambda_{\min }(P)\right)$.

Proof Construct an appropriate Lyapunov-Krasovskii function, $V(t)=\sum_{i=1}^{3} V_{i}(t)$, where $V_{1}(t)=\sum_{i=1}^{N} e_{i}^{T}(t) P_{i} e_{i}(t), V_{2}(t)=\sum_{i=1}^{N} \int_{t-\tau(t)}^{t} e_{i}^{T}(s) S_{i} e_{i}(s) d s, V_{3}(t)=\tau_{m} \sum_{i=N}^{N} \int_{-\tau_{m}}^{0} \int_{t+\vartheta}^{t} e_{i}^{T}(s) \times$ $Q_{i} e_{i}(s) d s d \vartheta$.

Then, combined with the proof of the relevant part of Theorem 1, we can get the following results:

$$
\begin{aligned}
\sum_{i=1}^{N} 2 e_{i}^{T}(t) P_{i} u_{i} \\
=\sum_{i=1}^{N} 2 e_{i}^{T}(t) P_{i}\left[-\left(K_{i}+\Delta K_{i}\right) e_{i}(t)-h_{1} \operatorname{sign}\left(e_{i}(t)\right) \Pi\right. \\
\quad-h_{2}\left(\int_{t-\tau(t)}^{t} e_{i}^{T}(s) S_{i} e_{i}(s) d s\right)^{\frac{1}{2}} \cdot \frac{e_{i}(t)}{\|e(t)\|^{2}} \\
\left.\quad-h_{3}\left(\tau_{m} \int_{-\tau_{m}}^{0} \int_{t+\vartheta}^{t} e_{i}^{T}(s) Q_{i} e_{i}(s) d s d \vartheta\right)^{\frac{1}{2}} \cdot \frac{e_{i}(t)}{\left\|e_{i}(t)\right\|^{2}}\right] \\
\leq-2 \sum_{i=1}^{N} e_{i}^{T}(t)\left(P_{i} K_{i}+K_{i}^{T} P_{i}+\varepsilon_{i 2} P_{i} H H^{T} P_{i}+\frac{1}{\varepsilon_{i 2}} E_{2}^{T} E_{2}\right)^{e_{i}(t)} \\
\quad-2 W \sum_{i=1}^{N}\left[\left(e_{i}^{T}(t) P_{i} e_{i}(t)\right)^{\frac{1}{2}}+\left(\int_{t-\tau(t)}^{t} e_{i}^{T}(s) S_{i} e_{i}(s) d s\right)^{\frac{1}{2}}\right.
\end{aligned}
$$




$$
\begin{gathered}
\left.+\left(\tau_{m} \int_{-\tau_{m}}^{0} \int_{t+\vartheta}^{t} e_{i}^{T}(s) Q_{i} e_{i}(s) d s d \vartheta\right)^{\frac{1}{2}}\right] \\
W=\min \left(h_{1} \frac{\lambda_{\min }\left(P_{i}\right)}{\lambda_{\max }^{\frac{1}{2}}(P)}, h_{2} \lambda_{\min }(P), h_{3} \lambda_{\min }(P)\right), \\
\dot{V}(e(t)) \leq \sum_{i=1}^{N} \xi_{i}^{T}(t) \Omega \xi_{i}(t)-2 W \sum_{i=1}^{N}\left[\left(e_{i}^{T}(t) P_{i} e_{i}(t)\right)^{\frac{1}{2}}+\left(\int_{t-\tau(t)}^{t} e_{i}^{T}(s) S_{i} e_{i}(s) d s\right)^{\frac{1}{2}}\right. \\
\left.+\left(\tau_{m} \int_{-\tau_{m}}^{0} \int_{t+\vartheta}^{t} e_{i}^{T}(s) Q_{i} e_{i}(s) d s d \vartheta\right)^{\frac{1}{2}}\right],
\end{gathered}
$$

where

$$
\Omega=\left[\begin{array}{ccc}
\Omega_{i 1} & 0 & 0 \\
* & \Omega_{i 2} & 0 \\
* & * & -Q_{i}
\end{array}\right],
$$

in which

$$
\begin{aligned}
\Omega_{i 1}= & P_{i} A+A^{T} P_{i}+\varepsilon_{i 1} P_{i} H H^{T} P_{i}+\frac{1}{\varepsilon_{i 1}} E_{1}^{T} E_{1}+2 L_{1} \lambda_{\max }\left(P_{i}\right) I+\varepsilon_{1}\left(\sum_{j=1}^{N} c_{i j}^{2}\right) P_{i} P_{i}^{T} \\
& +\frac{N L_{2}^{2}}{\varepsilon_{1}} I+\varepsilon_{2}\left(\sum_{j=1}^{N} d_{i j}^{2}\right) P_{i} P_{i}^{T}+S_{i}-2 P_{i} K_{i}+K_{i}^{T} P_{i}+\varepsilon_{i 2} P_{i} H H^{T} P_{i} \\
& +\frac{1}{\varepsilon_{i 2}} E_{2}^{T} E_{2}+\tau_{m}^{2} Q_{i}, \\
\Omega_{i 2}= & \frac{N L_{3}^{2}}{\varepsilon_{2}} I-(1-\dot{\tau}(t)) S_{i}, \quad U_{i}=P_{i} K_{i} .
\end{aligned}
$$

Using a proof method similar to that in Theorem 1, we can get

$$
\dot{V}(t) \leq-2 W V^{\frac{1}{2}}(t)
$$

Then we can know the error system (3) will synchronize in finite time $t^{*}$,

$$
t^{*}=\frac{V^{\frac{1}{2}}(0)}{W} .
$$

Remark 7 Let $U_{i}=P_{i} K_{i}$. In a computer simulation, the values of the matrices $\mathrm{P}$ and $\mathrm{U}$ can be calculated, and the value of the matrix $\mathrm{K}$ can be calculated using $K=P^{-1} U$.

Remark 8 In Theorem 3, we investigate the non-fragile control of a complex network system with uncertainty and time delay. In many practical applications, the parameters of the controller considerably vary. A non-fragile controller can be used to accurately estimate the upper bound of the time required for the system to reach the finite-time state. In Ref. [17], the author investigated a class of finite-time boundedness synchronization of stochastic complex networks. In Ref. [25], although the non-fragile controller is considered, the internal coupling relationship in the complex network model is linear, and only the matrix is uncertain. Clearly, this situation is a special form of the proposed model. 
In practical systems, uncertainty is ubiquitous. For systems without uncertainty, such as model (15), system instability may occur given the changes in controller parameters. Here, we investigate the finite-time synchronization problem of system (15) by using a non-fragile controller (21).

Corollary 4 For system (15), under Assumptions 1 and 2, if positive-definite symmetric matrices $K_{i}, P_{i}, S_{i}$ and $Q_{i}$ exist, so that

$$
\left[\begin{array}{ccc}
\Omega_{i 1} & 0 & 0 \\
* & \Omega_{i 2} & 0 \\
* & * & -Q_{i}
\end{array}\right]<0
$$

where

$$
\begin{aligned}
\Omega_{i 1}= & P_{i} A+A^{T} P_{i}+2 L_{1} \lambda_{\max }\left(P_{i}\right) I+\varepsilon_{1}\left(\sum_{j=1}^{N} c_{i j}^{2}\right) P_{i} P_{i}^{T}+\frac{N L_{2}^{2}}{\varepsilon_{1}} I+\varepsilon_{i 1} P_{i} H H^{T} P_{i} \\
& +\varepsilon_{2}\left(\sum_{j=1}^{N} d_{i j}^{2}\right) P_{i} P_{i}^{T}+S_{i}-2 P_{i} K_{i}+K_{i}^{T} P_{i}+\frac{1}{\varepsilon_{i 1}} E_{2}^{T} E_{2}+\tau_{m}^{2} Q_{i}, \\
\Omega_{i 2}= & \frac{N L_{3}^{2}}{\varepsilon_{2}} I-(1-\dot{\tau}(t)) S_{i}, \quad U_{i}=P_{i} K_{i} .
\end{aligned}
$$

Then the error system (16) can achieve finite-time synchronization, the time $t^{*}$ is

$$
t^{*}=\frac{V^{\frac{1}{2}}(0)}{W},
$$

where $W=\min \left(h_{1} \frac{\lambda_{\min }(P)}{\lambda_{\max }^{\frac{1}{2}}(P)}, h_{2} \lambda_{\min }(P), h_{3} \lambda_{\min }(P)\right)$.

In certain cases, the time delay in the system is a constant. For such a system, we can draw the following conclusion.

Corollary 5 If there exist matrices $P_{i}, K_{i}, S_{i}$ and $Q_{i}$, and the inequality (22) is existent,where $\dot{\tau}(t)=0$, then we consider the system (1) is synchronized at time $t^{*}$,

$$
t^{*}=\frac{V^{\frac{1}{2}}(0)}{W},
$$

where

$$
\begin{aligned}
& V(0)=\sum_{i=1}^{N}\left[e_{i}^{T}(0) P_{i} e_{i}(0)+\int_{-\tau}^{0} e_{i}^{T}(s) S_{i} e_{i}(s) d s+\int_{-\tau_{m}}^{0} \int_{\theta}^{0} e_{i}^{T}(s) Q_{i} e_{i}(s) d s d \theta\right] \\
& W=\min \left(h_{1} \frac{\lambda_{\min }(P)}{\lambda_{\max }^{\frac{1}{2}}(P)}, h_{2} \lambda_{\min }(P), h_{3} \lambda_{\min }(P)\right) .
\end{aligned}
$$




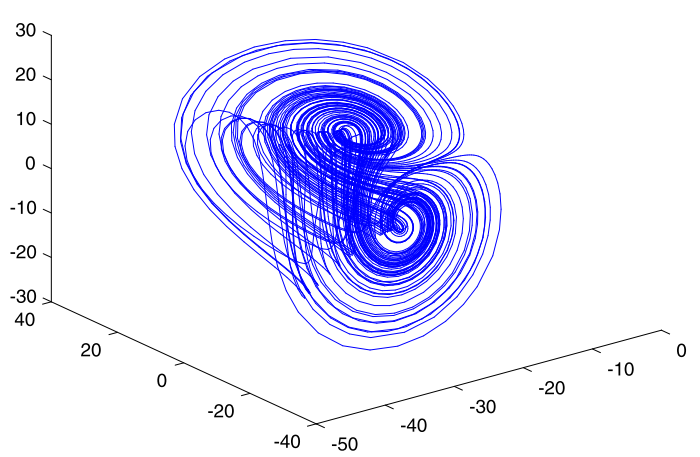

Figure 1 The chaotic trajectory of the system (27)

\section{Numerical simulation}

In this section, a numerical simulation is conducted to verify the main results of the theoretical analysis presented above. For a network model with six nodes, the internal dimension of each node is three, and the coupling between nodes, time-varying delay, and uncertainty are considered, and the state function of the system is described as

$$
\begin{aligned}
\dot{x}_{i}(t)= & (A+\Delta A(t)) x_{i}(t)+f\left(x_{i}(t)\right)+\sum_{j=1}^{N} c_{i j} g\left(x_{j}(t)\right)+\sum_{j=1}^{N} d_{i j} g\left(x_{j}(t\right. \\
& -\tau(t)))+u_{i}(t), \quad i=1,2, \ldots, N .
\end{aligned}
$$

Remark 9 Taking the epidemic disease spread model described in Remark 1 as an example, this paper investigates the finite-time synchronization problem of uncertain complex networks. Synchronization aims to ensure consistency of the relevant physical indicators of each patient with those of a healthy person.

The definition of $f(x)$ is given as follows, where

$$
f(x)=\left(\begin{array}{c}
-\frac{3}{8} x_{1}(t)+x_{2}(t) x_{3}(t) \\
\left(-x_{2}(t)+x_{3}(t)\right)(25-10 \cos (4.8 t)) \\
-x_{1}(t) x_{2}(t)-(15 \cos (4.8 t)+12) x_{2}(t)+(13.3-14 \cos (4.8 t)) x_{3}(t)
\end{array}\right) .
$$

The chaotic trajectory of the system (27) can be seen in Fig. 1.

Given the values of the matrix $A, C, D, H, E_{1}$, and other parameters in the system,

$$
\begin{aligned}
& A=\left[\begin{array}{ccc}
-2 & 1 & 0 \\
1 & -2 & 1 \\
0 & 1 & -2
\end{array}\right] ; \quad H=\left[\begin{array}{ccc}
0.1 & 0 & 1 \\
0 & 1 & 0 \\
1 & 0 & -1
\end{array}\right] ; \quad E_{1}=\left[\begin{array}{ccc}
-2 & 1 & 1 \\
0 & 0 & 0 \\
0 & 0 & 0
\end{array}\right] ; \\
& C=\left[\begin{array}{cccccc}
-2 & 1 & 1 & 0 & -1 & 1 \\
1 & -1 & 1 & -1 & 2 & -2 \\
1 & 1 & -2 & 2 & 0 & -2 \\
0 & 1 & -2 & 1 & -2 & 2 \\
-1 & 2 & 0 & -2 & 0 & 1 \\
1 & -1 & -1 & 2 & 1 & -2
\end{array}\right] ; \quad D=\left[\begin{array}{cccccc}
-2 & 1 & 1 & 0 & -1 & 1 \\
1 & -2 & 1 & 1 & 2 & -3 \\
1 & 1 & -2 & 2 & 0 & -2 \\
1 & 1 & -2 & 0 & -2 & 2 \\
0 & 2 & 1 & -2 & -2 & 1 \\
1 & -1 & -1 & 2 & 1 & -2
\end{array}\right] ;
\end{aligned}
$$




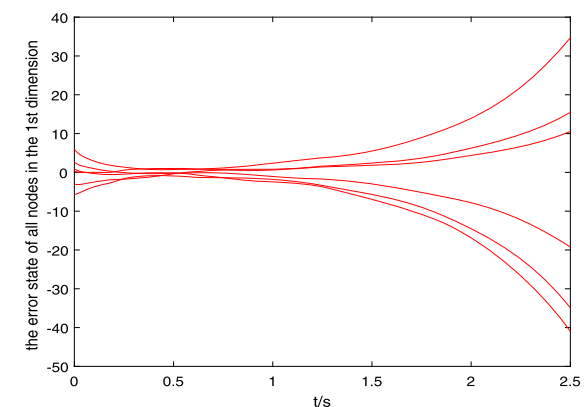

(a)

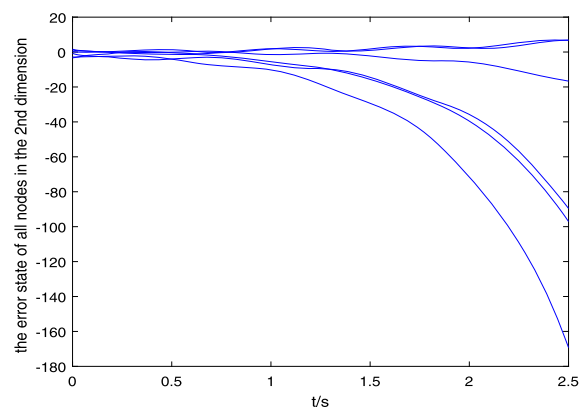

(b)

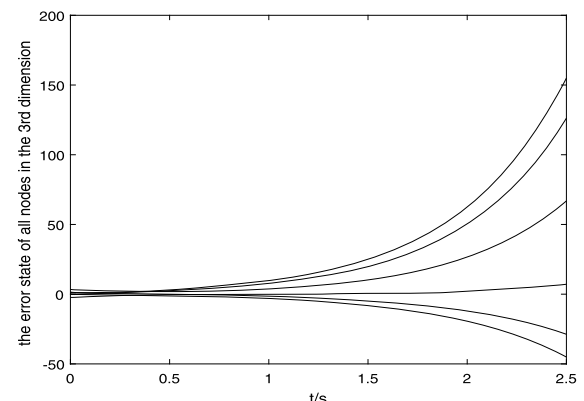

(c)

Figure 2 The state of all nodes in different dimensions without controller

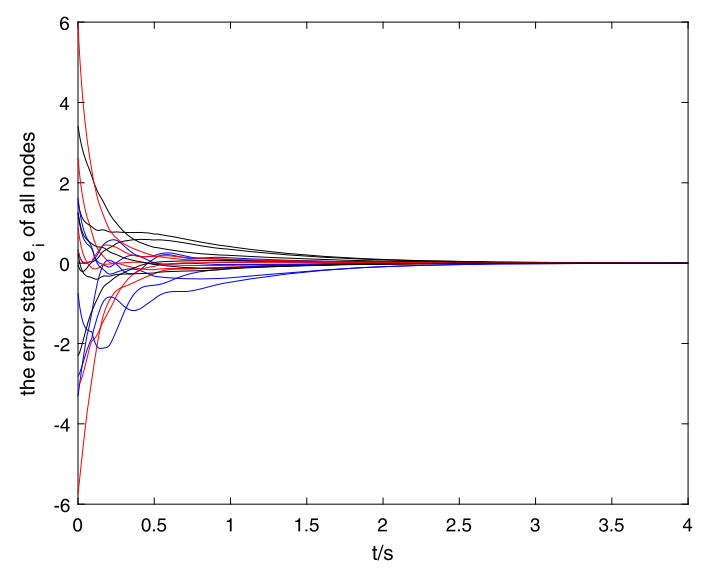

Figure 3 The error state $e_{i}$ of all nodes when $h_{1}=3, h_{2}=3, h_{3}=4, h_{4}=5$ and $\theta=0.3$

$F(t)=\operatorname{diag}([\sin (t), \cos (2 t), \sin (2 t)])$.

The nonlinear vector-valued function in the complex network are described by $g(x)=$ $\tanh (x), \tau(t)=1 / 2-1 / 3 e^{-t}$. According to Theorem 1 and the LMI toolbox in MATLAB, $P_{i}$, $S_{i}$ and $Q_{i}$ satisfying condition (5) of Theorem 1 can be obtained, and the motion trajectory of the error system without and with the controller is shown in Figs. 2, 3, 4, respectively.

According to Fig. 3, the system can achieve fast synchronization in a short time by using the controller (4) when $\theta=0.3$. At this point, the maximum time delay allowed by the 


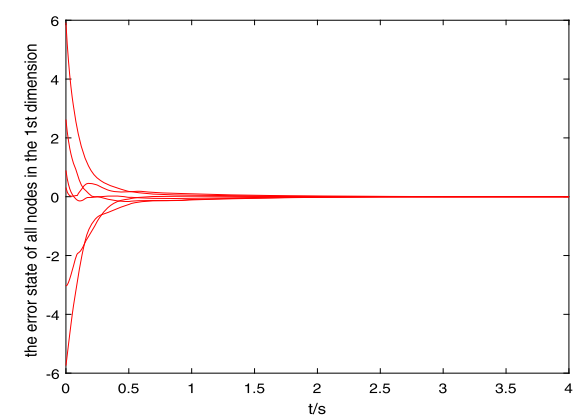

(a)

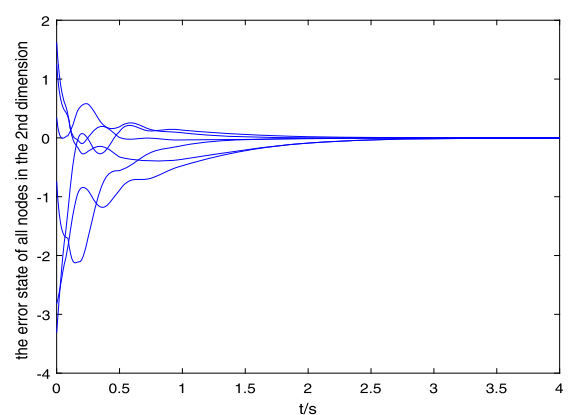

(b)

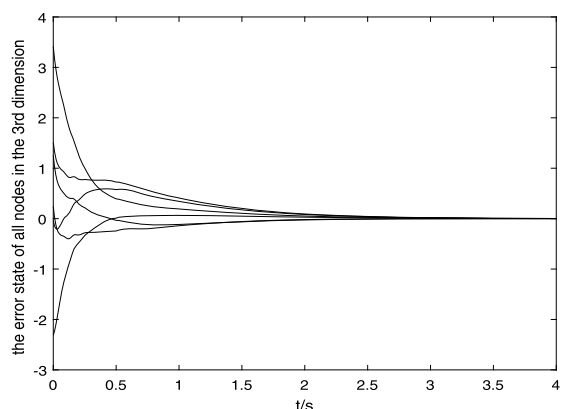

(c)

Figure 4 The error state of all nodes in different dimensions with the controller (4)

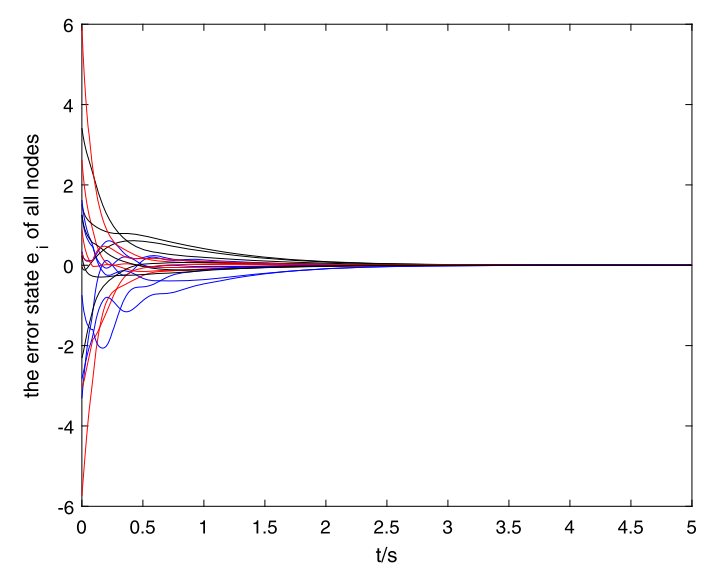

Figure 5 The error state $e_{i}$ of all nodes when $h_{1}=3, h_{2}=3, h_{3}=4, h_{4}=5$ and $\theta=0.8$

system is $0.692 \mathrm{~s}$. In Fig. 5, $\theta=0.8$. Hence, the required synchronization time for the system increases compared with Fig. 3.

Figure 6 shows a faster convergence rate than Fig. 3. Therefore, the required synchronization time for the system can be shortened by appropriately increasing the value of each parameter $h_{i}$ in the controller.

\section{Conclusion}

In this study, a finite-time synchronization of uncertain nonlinear complex dynamic networks with time-varying delay is investigated. In this network model, the uncertainty and 


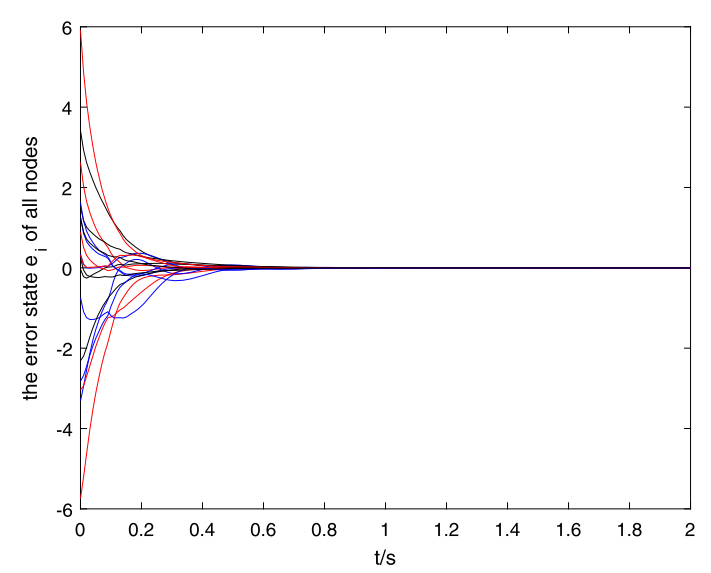

Figure 6 The error state $e_{i}$ of all nodes when $h_{1}=8, h_{2}=6, h_{3}=8, h_{4}=10$ and $\theta=0.3$

coupling time delay of the system are considered simultaneously where the uncertainties satisfy the matching conditions. An appropriate feedback controller is designed by constructing the appropriate Lyapunov-Krasovkii function and matrix inequality, and sufficient conditions are obtained for the system to achieve finite-time synchronization. The feasibility of the theorem is verified through a computer simulation.

The controller was designed in this paper to act continuously on each node to guarantee the finite-time synchronization of the system. However, resource utilization must still be improved. Therefore, the addition of an event-triggered mechanism will be considered in future work. The controller and the moment will only be updated when the trigger condition is met to save communication resources and reduce the frequency of controller updates. In addition, the method used in this paper is suitable for a moderate number of network nodes; otherwise, this method will affect the speed of solving matrix inequality in simulation. Hence, another direction that will be considered in the future is the acceleration of the matrix solution when the number of network nodes in the system is large.

Acknowledgements

The authors thank the editor and the anonymous reviewers for their insightful comments and suggestions that improved the quality and presentation of this paper.

Funding

This work was jointly supported by the National Natural Science Foundation of China (Grant No. 11972156) and Natural Science Foundation of Hunan Province (Grant No. 2017JJ4004).

Availability of data and materials

The data used to support the findings of this study are available from the corresponding author upon request.

Competing interests

The authors declare that there is no conflict of interests regarding the publication of this paper.

Authors' contributions

The two authors contributed equally to the writing of this paper. Furthermore, both authors carefully read and approved the final manuscript.

\section{Publisher's Note}

Springer Nature remains neutral with regard to jurisdictional claims in published maps and institutional affiliations. 


\section{References}

1. Guerrero, G., Langa, J.A., Suarez, A.: Attracting complex networks. Compl. Netw. Dyn. 693, 309-327 (2016)

2. D'Arcangelis, A.M., Rotundo, G.: Complex Networks in Finance. In: Complex Networks and Dynamics, vol. 683, pp. 209-235. Springer, Berlin (2016)

3. Kumari, S., Singh, A.: Modeling of data communication networks using dynamic. Compl. Netw. Perform. Stud. 693 29-40 (2016)

4. Sorrentino, F., Pecora, L.M., Hagerstrom, A.M., et al.: Complete characterization of the stability of cluster synchronization in complex dynamical networks. Sci. Adv. 2(4), e1501737-e1501737 (2016)

5. Xiang, W., Jun-Chan, Z., Chun-Hua, H.U., et al.: Generalized synchronization and system parameters identification between two different complex networks. Acta Autom. Sin. 43(4), 595-603 (2017)

6. Wang, X., She, K., Zhong, S., et al.: Pinning cluster synchronization of delayed complex dynamical networks with nonidentical nodes and impulsive effects. Nonlinear Dyn. 88(4), 2771-2782 (2017)

7. Yang, H., Shu, L., Zhong, S.: Pinning lag synchronization of complex dynamical networks with known state time-delay and unknown channel time-delay. Nonlinear Dyn. 89(3), 1793-1802 (2017)

8. Luo, Y., Shu, L.: Exponential synchronization of nonlinearly coupled complex dynamic networks with time-varying coupling delays. Complexity 2017, Article ID 7850958 (2017)

9. Zhang, W., Li, C., He, X., et al.: Finite-time synchronization of complex networks with non-identical nodes and impulsive disturbances. Mod. Phys. Lett. B 32(1), 1850002 (2017)

10. Selvaraj, P., Sakthivel, R., Kwon, O.M.: Synchronization of fractional-order complex dynamical network with random coupling delay, actuator faults and saturation. Nonlinear Dyn. 94, 3101-3116 (2018)

11. Sun, Y., Ma, Z., Liu, F., et al.: Theoretical analysis of synchronization in delayed complex dynamical networks with discontinuous coupling. Nonlinear Dyn. 86(1), 489-499 (2016)

12. Ma, Y., Ma, N., Chen, L.: Synchronization criteria for singular complex networks with Markovian jump and time-varying delays via pinning control. Nonlinear Anal. Hybrid Syst. 29, 85-99 (2018)

13. Wang, X., Liu, X., Zhong, S.: Pinning impulsive synchronization of complex dynamic networks with various time-varying delay sizes. Nonlinear Anal. Hybrid Syst. 26, 307-318 (2017)

14. Li, J., Jiang, H., Hu, C., Yu, J.: Analysis and discontinuous control for finite-time synchronization of delayed complex dynamic networks. Chaos Solitons Fractals 114, 291-305 (2018)

15. Zhang, D., Shen, Y., Mei, J.: Finite-time synchronization of multi-layer nonlinear coupled complex networks via intermittent feedback control. Neurocomputing 225, 129-138 (2016)

16. Li, N., Feng, J., Zhao, Y.: Finite-Time Synchronization for Nonlinearly Coupled Networks with Time-Varying Delay. Control and Decision Conference. IEEE Press, New York (2016)

17. Sakthivel, R.: Sakthivel, R. et al.: Finite-time nonfragile synchronization of stochastic complex dynamic networks with semi-Markov switching outer coupling. Complexity 2018, Article ID 8546304 (2018)

18. Xiao, F., Gan, Q.: Finite-time synchronization of delayed complex dynamical network via pinning control. Adv. Differ. Equ. 2017, 345 (2017)

19. Ramalingam, S., et al.: Observer-based robust synchronization of fractional-order multi-weighed complex dynamic networks. Nonlinear Dyn. 98, 1231-1246 (2019)

20. Ali, M.S., Yogambigai, J.: Finite-time robust stochastic synchronization of uncertain Markovian complex dynamical networks with mixed time-varying delays and reaction-diffusion terms via impulsive control. J. Franklin Inst. 354(5), 2415-2436 (2017)

21. Mei, J., Jiang, M., Wang, J.: Finite-time structure identification and synchronization of drive-response systems with uncertain parameter. Commun. Nonlinear Sci. Numer. Simul. 18(4), 999-1015 (2013)

22. Zhao, H., Zheng, M., Li, S., et al.: New results on finite-time parameter identification and synchronization of uncertain complex dynamical networks with perturbation. Mod. Phys. Lett. B 2018, 1850112 (2018)

23. Wang, H., Ye, J., Miao, Z., Jonckheere, A.: Robust finite-time chaos synchronization of time-delay chaotic systems and its application in secure communication. Trans. Inst. Meas. Control 40(4), 1177-1187 (2018)

24. Mobayen, S., Ma, J.: Robust finite-time composite nonlinear feedback control for synchronization of uncertain chaotic systems with nonlinearly and time-delay. Chaos Solitons Fractals 114, 46-54 (2018)

25. Yang, X., Ho, D., Lu, J., et al.: Finite-time cluster synchronization of T-S fuzzy complex networks with discontinuous subsystems and random coupling delays. IEEE Trans. Fuzzy Syst. 23, 6 (2015)

26. Zhang, M., Han, M.: Finite-time synchronization of uncertain complex networks with nonidentical nodes based on a special unilateral coupling control. Adv. Neur. Netw. 10262, 161-168 (2017)

27. Zhao, H., Li, L., Peng, H., et al.: Finite-time robust synchronization of memristive neural network with perturbation. Neural Process. Lett. 47, 2 (2017)

28. Cai, Z., Huang, L., Zhang, L.: Improved switching controllers for finite-time synchronization of delayed neural networks with discontinuous activations. J. Franklin Inst. 354(15), 6692-6723 (2017)

29. Zhao, H., Cai, G.: Exponential synchronization of complex delayed dynamical networks with uncertain parameters via intermittent control. In: Advances in Neural Networks-ISNN, vol. 9377, pp. 91-98. Springer, Berlin (2015)

30. Kaviarasan, B., Sakthivel, R., Lim, Y.: Synchronization of complex dynamical networks with uncertain inner coupling and successive delays based on passivity theory. Neurocomputing 196, 127-138 (2016)

31. Wu, X., Lu, H.: Outer synchronization of uncertain general complex delayed networks with adaptive coupling. Neurocomputing 82, 157-166 (2012)

32. Wang, T., Zhou, W., Zhao, S.: Robust synchronization for stochastic delayed complex networks with switching topology and unmodeled dynamics via adaptive control approach. Commun. Nonlinear Sci. Numer. Simul. 18(8), 2097-2106 (2013)

33. Shi, H., Sun, Y., Miao, L., et al.: Outer synchronization of uncertain complex delayed networks with noise coupling Nonlinear Dyn. 85(4), 2437-2448 (2016)

34. Zhang, C., Wang, X., Wang, C., et al.: Synchronization of uncertain complex networks with time-varying node delay and multiple time-varying coupling delays. Asian J. Control 20(1), 186-195 (2018)

35. Cui, W., Sun, S., Fang, J.A., et al.: Finite-time synchronization of Markovian jump complex networks with partially unknown transition rates. J. Shenzhen Univ. 351(5), 2543-2561 (2014) 
36. Liu, M., Jiang, H., Hu, C.: Finite-time synchronization of delayed dynamical networks via aperiodically intermittent control. J. Franklin Inst. 354, 5374-5397 (2017)

37. Liu, M., Wu, J., Sun, Y.Z:: Adaptive finite-time outer synchronization between two complex dynamical networks with noise perturbation. Nonlinear Dyn. 89(49), 1-11 (2017)

38. Li, D., Cao, J.: Finite-time synchronization of coupled networks with one single time-varying delay coupling. Neurocomputing 166, 265-270 (2015)

39. Landis, J.G., Perlmutter, D.D.: Stability of time-delay systems. AlChE J. 18(2), 380-384 (1972)

40. Zuo, Z., Lin, T.: Distributed robust finite-time nonlinear consensus protocols for multi-agent systems. Int. J. Syst. Sci. 47(6), 1-10 (2014)

41. Tang, Y.: Terminal sliding mode control for rigid robots. Automatica 34(1), 51-56 (1998)

42. Wu, M., He, Y., She, J.H.: Stability Analysis and Robust Control of Time-Delay Systems (2010) https://doi.org/10.1007/978-3-642-03037-6

43. Syed, A.M., Yogambigai, J.: Extended dissipative synchronization of complex dynamical networks with additive time-varying delay and discrete-time information. J. Comput. Appl. Math. 348, 328-341 (2019)

44. Yu, R., et al.: Synchronization criterion of complex networks with time-delay under mixed topologies. Neurocomputing 295, 8-16 (2018)

45. Shi, L., Chen, G., Zhong, S.: Outer synchronization of a class of mixed delayed complex networks based on pinning control. Adv. Differ. Equ. 2018, 330 (2018)

46. Wang, L., Song, Q., et al.: Synchronization of two nonidentical complex-valued neural networks with leakage delay and time-varying delays. Neurocomputing 356, 52-59 (2019)

47. Huang, X., Ma, Y.: Finite-time $H_{\infty}$ sampled-data synchronization for Markovian jump complex networks with time-varying delays. Neurocomputing 296, 82-99 (2018)

48. Min, H., Meng, Z., Tie, Q., et al.: UCFTS: a unilateral coupling finite-time synchronization scheme for complex networks. IEEE Trans. Neural Netw. 30(1), 255-268 (2019)

49. Cheng, L., Yang, Y., Li, L., Sui, X.: Finite-time hybrid projective synchronization of the drive-response complex networks with distributed-delay via adaptive intermittent control. Physica A 200, 273-286 (2018)

\section{Submit your manuscript to a SpringerOpen ${ }^{\circ}$ journal and benefit from:}

- Convenient online submission

- Rigorous peer review

- Open access: articles freely available online

- High visibility within the field

- Retaining the copyright to your article

Submit your next manuscript at $\gg$ springeropen.com 